\title{
No evidence of an increased mortality risk associated with low levels of glycated haemoglobin in a non-diabetic UK population
}

\author{
R. Pfister • S. J. Sharp • R. Luben • K.-T. Khaw • \\ N. J. Wareham
}

Received: 11 October 2010 /Accepted: 17 March 2011/Published online: 17 May 2011

(C) Springer-Verlag 2011

\begin{abstract}
Aims/hypothesis There is debate about increased mortality risk associated with low levels of glycaemia. To address this issue, we examined the shape of the risk relationship between glycated haemoglobin and mortality in a UK population.

Methods In 17,196 men and women aged 39-82 years participating in the European Prospective Investigation into Cancer and Nutrition (EPIC) Study in Norfolk without known diabetes or cardiovascular disease, we estimated HRs for total and cause-specific mortality comparing categories of glycated haemoglobin $(<4.5 \%, 4.5 \%$ to $<5.0 \%, 5.0 \%$ to $<5.5 \%$ [reference], $5.5 \%$ to $<6.0 \%, 6.0 \%$ to $<6.5 \%$, and $\geq 6.5 \%$ ) using Cox regression.

Results During a mean $( \pm \mathrm{SD})$ follow-up of $11.2( \pm 2.1)$ years 1,953 participants died. The HR for all-cause mortality increased with categories of increasing glycated haemoglobin in adjusted analyses (HR 0.94 [95\% CI $0.72-$ 1.22], 0.99 [0.86-1.13], 1.00 [0.92-1.08], 1.10 [1.02-1.19], 1.29 [1.14-1.46] and 1.45 [1.16-1.80]). Spline regression suggested no increased risk at the low end of the

R. Pfister · S. J. Sharp · N. J. Wareham $(\bowtie)$

Medical Research Council Epidemiology Unit,

Institute of Metabolic Science, Addenbrooke's Hospital,

Box 285, Hills Road,

Cambridge CB2 0QQ, UK

e-mail: nick.wareham@mrc-epid.cam.ac.uk

R. Pfister

Department III of Internal Medicine,

Heart Centre of the University of Cologne,

Cologne, Germany

R. Luben $\cdot$ K.-T. Khaw

Department of Public Health and Primary Care, Institute of Public Health, University of Cambridge,

Cambridge, UK
\end{abstract}

distribution. Indeed, the HR for all-cause mortality was virtually constant in the low range and only started to rise when the level was approximately $5.5 \%$. There were similar associations of glycated haemoglobin with cause-specific mortality, with the strongest association being seen for cardiovascular mortality.

Conclusions/interpretation Our findings in a large nondiabetic population do not support the concern about increased mortality risk with low glycated haemoglobin. Differences in population characteristics might explain contrary results of earlier studies and need further exploration.

Keywords $\mathrm{HbA}_{1 \mathrm{c}} \cdot$ Low glycaemia $\cdot$ Mortality

\begin{tabular}{|c|c|}
\hline \multicolumn{2}{|c|}{ Abbreviations } \\
\hline ACCORD & $\begin{array}{l}\text { Action to Control Cardiovascular Risk in } \\
\text { Diabetes }\end{array}$ \\
\hline ARIC & Atherosclerosis Risk in Communities \\
\hline ALT & Alanine aminotransferase \\
\hline AST & Aspartate aminotransferase \\
\hline EPIC & $\begin{array}{l}\text { European Prospective Investigation into } \\
\text { Cancer and Nutrition }\end{array}$ \\
\hline NHANES & $\begin{array}{l}\text { National Health and Nutrition Examination } \\
\text { Survey }\end{array}$ \\
\hline
\end{tabular}

Introduction

Glycated haemoglobin is recommended for the diagnosis and monitoring of non-diabetic hyperglycaemia and diabetes mellitus [1]. A key premise for this recommendation is the documented evidence that glycated haemoglobin predicts the risk of diabetes and related complications. 
Two recent analyses of the Atherosclerosis Risk in Communities (ARIC) Study and the National Health and Nutrition Examination Survey (NHANES) described a significantly increased risk of low levels of glycated haemoglobin for all-cause mortality in non-diabetic populations $[2,3]$. This adds to the existing debate on a potentially increased health risk of low-normal glycaemia, which was initiated by the results of a recent trial in patients with advanced diabetes mellitus and by observational evidence from a pharmacosurveillance study in patients with diabetes mellitus $[4,5]$. The findings of the ARIC study and NHANES cannot be explained by diabetesspecific effects or glucose-lowering treatment and thus, if they represent a true adverse association of low glycated haemoglobin levels with risk, then these findings would have clinical implications. If low-normal glycaemia itself is the reason for the adverse health outcome, our understanding of the impact of glycaemia on risk would be altered, as low glycaemia is intuitively assumed to be beneficial. Alternatively, if non-glycaemic determinants of glycated haemoglobin were the reason for the association with adverse health outcomes, putative mechanisms underlying this association and potential effects on diagnostic accuracy of glycated haemoglobin for diabetes and high-risk states need to be explored.

Most previous studies examining the association of glycated haemoglobin with all-cause mortality in nondiabetic populations have had limited statistical power to test the nature of the association at the low end of the distribution [6-9]. Therefore, in order to test the shape of association and explore potential underlying mechanisms, we analysed the association of glycated haemoglobin with all-cause and cause-specific mortality in men and women in a UK population-based study. We have previously reported findings from a baseline survey of the European Prospective Investigation into Cancer and Nutrition (EPIC) study in Norfolk conducted 1993-1997 [10, 11]. We report now an extended analysis, with almost doubled follow-up time, that additionally includes an independent measure of glycated haemoglobin obtained in participants attending a second examination in 1998-2000. Thus, sample size and number of events were substantially increased, which allows an accurate risk estimation, particularly for the low nondiabetic range of glycated haemoglobin.

\section{Methods}

EPIC-Norfolk is a prospective cohort study in which men and women aged 39 to 79 years were recruited from general practices in the Norfolk region, England, UK. Full details of the population are reported elsewhere by Day et al. [12]. In brief, between 1993 and 1997, 25,639 individuals underwent a baseline health examination that included completion of a general health questionnaire. Questions were asked about medical history including diabetes, myocardial infarction and stroke as well as family history of myocardial infarction and stroke, smoking status, medication intake, alcohol consumption and physical activity. During a clinical visit anthropometric data and blood pressure were assessed. Non-fasting blood samples were collected, and lipid status was assessed. Details of the measurement of baseline characteristics have been reported previously by Khaw et al. [13]. Starting in 1995, when funding became available, glycated haemoglobin levels were measured on fresh EDTA blood samples using highperformance liquid chromatography (Bio-Rad Diamat or Bio-Rad Variant II Automated Glycated Haemoglobin Analyzer; Bio-Rad Laboratories, Hemel Hempstead, UK). From 1998 to 2000 the cohort was invited for a second examination in which most measurements performed at the baseline health check were repeated. Glycated haemoglobin was measured in all 15,786 individuals attending the second health check using the same method as for the baseline measurements. To maximise sample size and follow-up time, we included all participants with results available for glycated haemoglobin from the baseline survey $(n=11,147)$ and combined them with participants of the second health check (assigned now as baseline; all covariates of these participants included in the analysis were assessed during the second health check except liver indices), who did not have glycated haemoglobin measured at the baseline survey $(n=8,606)$. In 2010 additional laboratory variables, including the liver damage tests aspartate aminotransferase (AST) and alanine aminotransferase (ALT), were measured on stored serum samples which were available for about 19,500 participants of the baseline health examination.

We report results of follow-up to 30 June 2009, a mean of 11.2 years. All EPIC-Norfolk participants were flagged for death certification at the Office for National Statistics, with virtually complete vital status for all participants. A cardiovascular death was defined according to ICD-9 (www.icd9data.com/2007/Volume1/240-279/250-259/250/ default.htm) or ICD-10 (www.who.int/classifications/icd/ en/) codes: 400-448 (ICD-9) or I10-I79 (ICD-10) listed as the underlying cause of death; cancer was ICD-9 codes 140-208 or ICD-10 codes C00-C97.

Baseline diabetes mellitus status was ascertained by means of self-report of diabetes, diabetic diet, diabetesspecific medication or diabetes-specific medication brought to the clinical visit. Baseline cardiovascular disease was defined by self-reported myocardial infarction or stroke, and if the second health check was considered the baseline examination, additionally by hospital admission for cardiovascular disease occurring between the first and second 
health check. Hospitals were linked to the East Norfolk Health Authority database, which identifies all hospital contacts for Norfolk residents throughout England and Wales. Of all identified hospitalisations in this cohort, 93\% occurred within Norfolk, i.e. 7\% occurred outside Norfolk but within England or Wales, suggesting that hospitalisations occurring outside England or Wales, which were missed in our analysis, might be rare and not influence results.

We excluded individuals with known diabetes mellitus $(n=621)$ and known cardiovascular disease $(n=1,015)$ as these conditions, if clinically known, may lead to interventions influencing both glycated haemoglobin concentrations and outcome, or missing baseline data for any of these variables $(n=921)$. The final data set included 17,196 people.

\section{Statistical analysis}

We divided the cohort into six categories on the basis of their baseline levels of glycated haemoglobin: $<4.5 \%, 4.5 \%$ to $<5.0 \%, 5.0 \%$ to $<5.5 \%, 5.5 \%$ to $<6.0 \%, 6.0 \%$ to $<6.5 \%$, and $\geq 6.5 \%$. We examined risk factor distribution by glycated haemoglobin category, using linear regression, logistic regression and a non-parametric test for ordered groups to test for trend across categories. We used a Cox proportional hazards model to determine the independent contribution of glycated haemoglobin to all-cause and cause-specific mortality. Covariates for adjustment were selected by fitting a Cox model, initially including all variables of Table 1 (except ALT and AST which were available only for about 12,000 participants) with a significant $(p<0.05)$ association in univariate analysis and then removing any covariates with a $p$ value for association of $>0.1$. Accordingly, multivariable analyses were adjusted for age (as the underlying timescale), sex, systolic blood pressure, total cholesterol, smoking status, waist-to-hipratio, alcohol consumption and physical activity. CIs were calculated using quasi-standard errors, which are based both on the variances of the estimated log HRs and the covariances between them [14].

To assess the continuous association between glycated haemoglobin and mortality, we generated piecewise linear splines with knots corresponding to cut-off points used in this study ( $4.5 \%, 5 \%, 5.5 \%$ and $6 \%$ ); we also implemented restricted cubic splines to obtain a smoother fit to the data with knots at the 5th, 25th, 75th and 95th percentiles of glycated haemoglobin. Both models were centred at the median $(5.3 \%)$ and the plot was truncated at the 2.5 th and 97.5th percentiles of glycated haemoglobin $(4.2 \%$ and $6.4 \%$, respectively). Statistical departure from linearity was tested by calculating the difference in log likelihood for modelling categories of glycated haemoglobin as a continuous term against categories of glycated haemoglobin. We also tested linearity for categories of decile of glycated haemoglobin.

We tested for interaction between sex and glycated haemoglobin. Cox regression analysis was repeated further adjusting for examination year to account for possible drift in glycated haemoglobin assay and excluding those with death occurring during the initial 3 years of follow-up to account for potential influence of prevalent disease on levels of glycated haemoglobin levels.

All the analyses were completed using statistical software programs (Stata version 11.0 [Stata Corp, College Station, TX, USA] and R version 2.11.0 [http://www.rproject.org]).

The EPIC-Norfolk study was approved by the Norfolk Local Research Ethics Committee, and participants gave signed informed consent at each contact.

\section{Results}

Baseline characteristics of the study population overall and by glycated haemoglobin category are given in Table 1. Of the total population, $99.6 \%$ were white Europeans. The mean age was 59.2 years, mean glycated haemoglobin level was 5.3\% and 3,742 (22\%) individuals had glycated haemoglobin levels below 5\%. All measured cardiovascular risk factors significantly increased or were more frequent with categories of increasing glycated haemoglobin levels. Variables of liver damage (ALT and AST) increased with categories of increasing glycated haemoglobin.

A total of 1,953 people died during a mean follow-up of $11.2 \pm 2.1$ years. There was no significant interaction between sex and glycated haemoglobin for any of the outcomes $(p>0.15)$, so we present sex-combined results only. Table 2 shows the estimated HRs for death from all causes according to the baseline category of glycated haemoglobin adjusted for age and sex. The risk for allcause death increased throughout the whole range of glycated haemoglobin concentration. After adjustment for risk factors, we observed a continuous increase in the risk of all-cause mortality with categories of increasing glycated haemoglobin (Fig. 1a). There was no statistically significant evidence for a departure from linearity $(p=0.65)$. Results were closely similar in the sex-stratified analysis, after adjusting for examination year and after exclusion of all people who died during the first 3 years of follow-up (data not shown). Piecewise-linear and restricted-cubic spline regression suggested that the HR for all-cause mortality (relative to a level of 5.3\%) is virtually constant for the low range of glycated haemoglobin and starts to increase at 


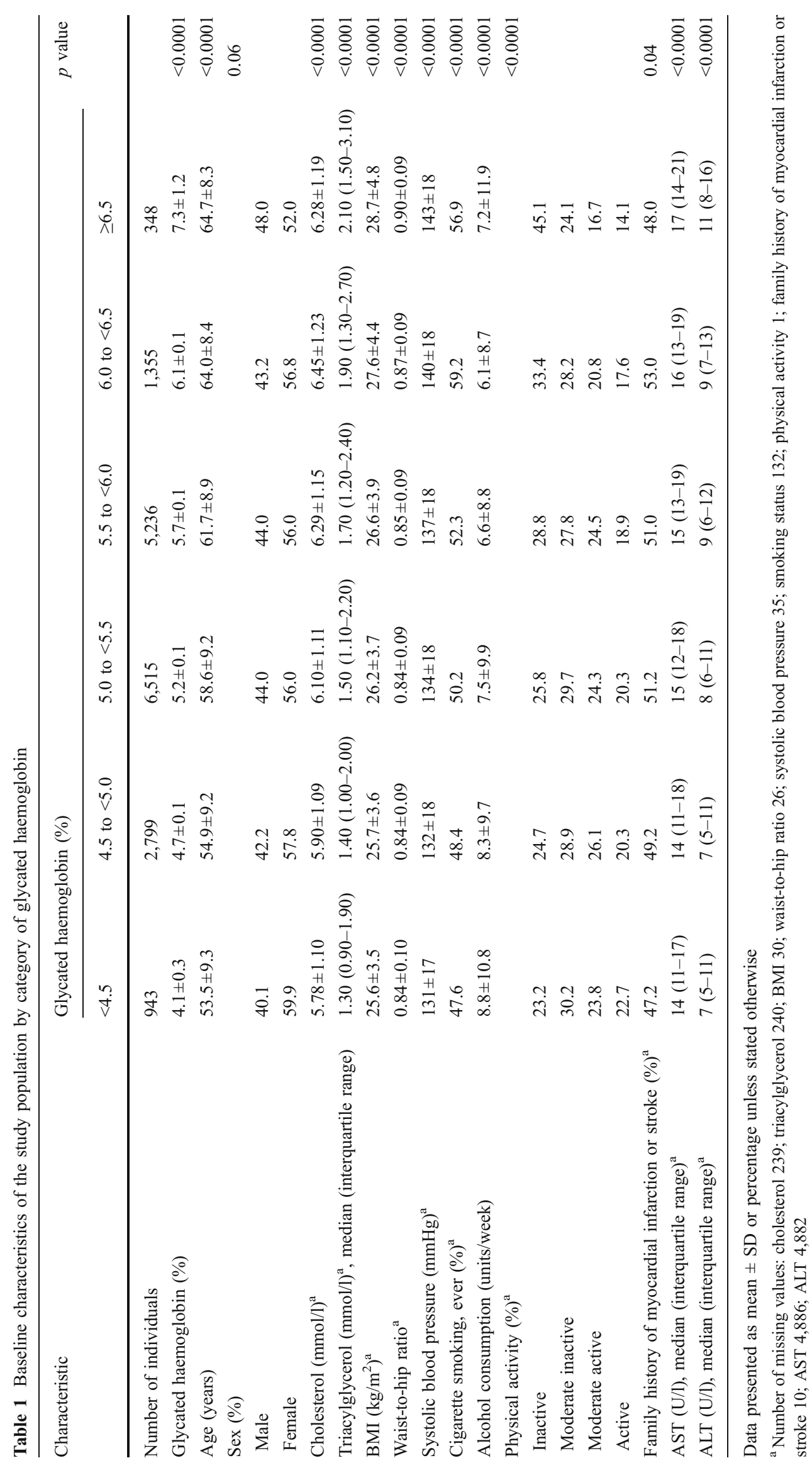



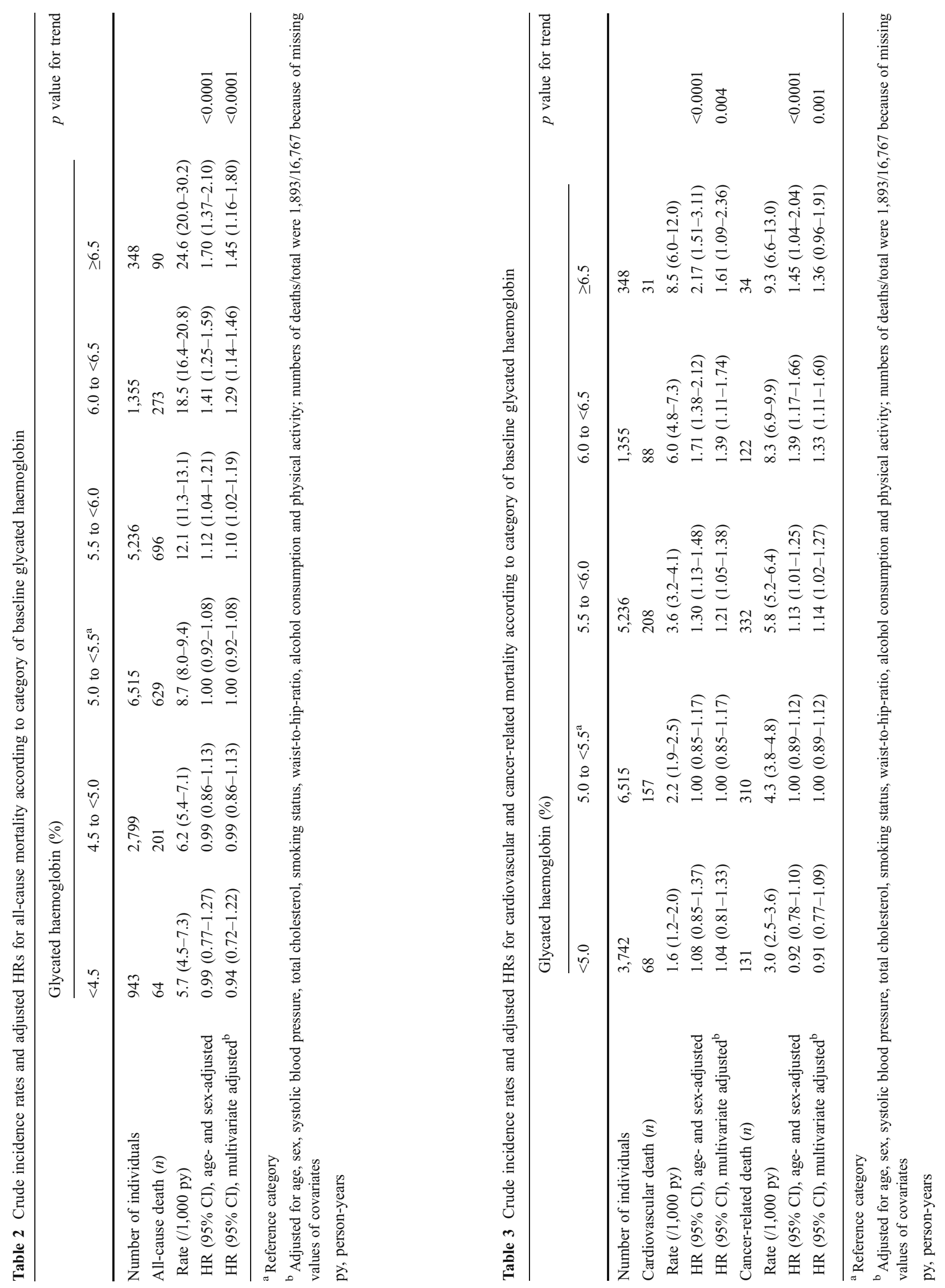

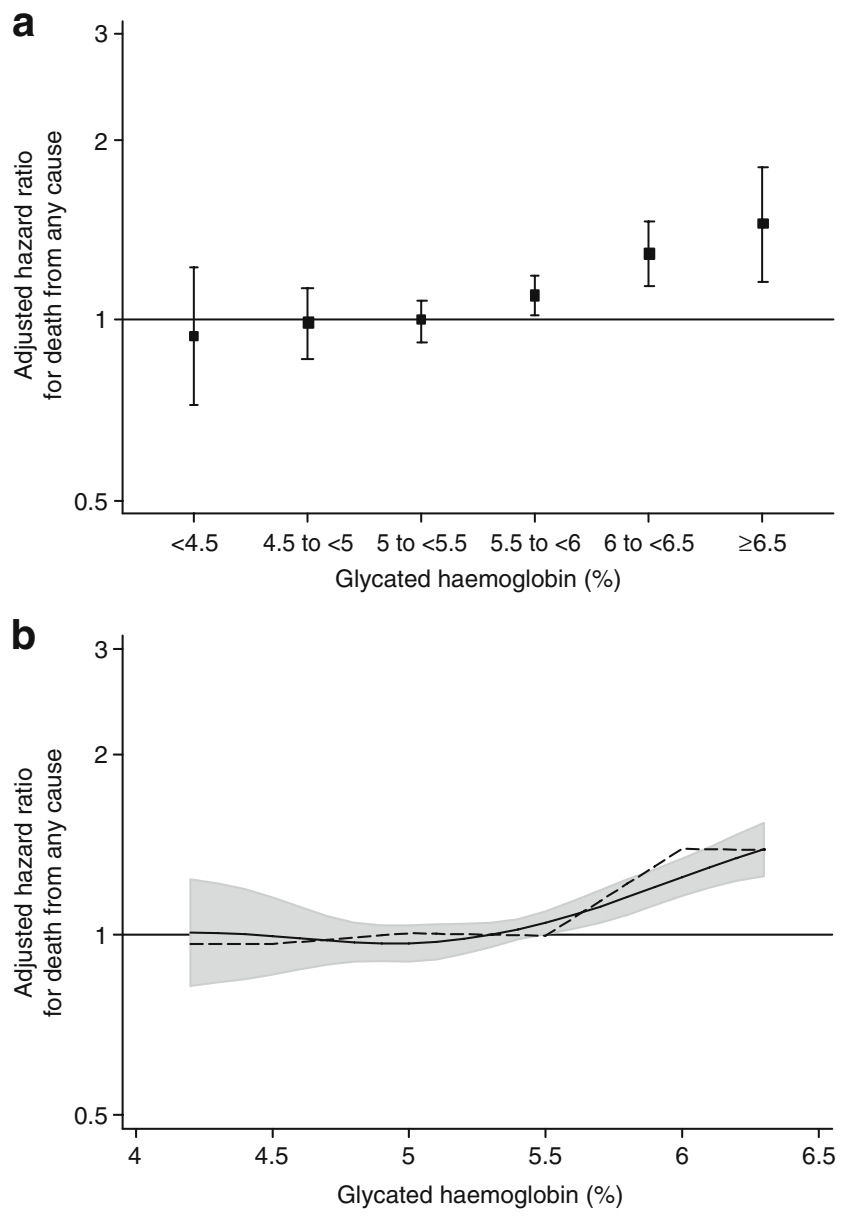

Fig. 1 Adjusted HRs for all-cause mortality by (a) categories of baseline glycated haemoglobin and (b) piecewise-linear-spline (dashed line) and restricted-cubic-spline regression (black line, shaded area is the $95 \% \mathrm{CI}$ )

approximately $5.5 \%$, with no suggestion of an increased risk at the low end of the distribution (Fig. 1b).

As a result of the low number of events in the lower categories of glycated haemoglobin for analyses of causespecific mortality, we combined the two lowest categories to one category including levels below 5\% (Table 3). Overall, the estimated HRs for cardiovascular and cancerrelated mortality increased with categories of increasing glycated haemoglobin levels in multivariate analyses. The association of glycated haemoglobin was largest for cardiovascular mortality, although the association with cancer-related mortality showed a similar trend.

\section{Discussion}

We observed a continuous increase in the risk for allcause mortality throughout the whole range of glycated haemoglobin values in a non-diabetic UK population, with suggestion of a 'basement' to the graded relation for the low range. There was a similar pattern of association for cardiovascular and cancer-related mortality, with the strongest association being observed for cardiovascular mortality.

The shape of the association between a risk factor such as glycaemia and health outcome has major implications for the recommendation of targets for interventions. There is broad evidence for hyperglycaemia as a risk factor for cardiovascular disease $[15,16]$, extended very recently by results of a large collaborative meta-analysis of 102 studies on fasting glucose concentrations [17], but data on the low range of glycaemia are inconsistent. In addition to the ARIC study and NHANES, four earlier studies reported mortality risk for categories of glycated haemoglobin in non-diabetic populations, with one study suggesting a potentially increased risk [8] and three studies suggesting no increased risk with low levels of glycated haemoglobin $[6,7,9]$. However, low numbers of events, particularly in the low range of glycated haemoglobin (below $~ 5.0 \%$ ), limit an accurate risk estimation in these studies. In contrast to the ARIC and NHANES results, our findings suggest that there is no lower threshold of glycated haemoglobin associated with increased risk for mortality.

We cannot distinguish in our analysis between a shallow linear increase in risk across the entirety of the distribution of glycated haemoglobin and a very flat relationship below a threshold above which risk increases linearly. In general it is difficult to distinguish between threshold relationships and linear ones and to identify the exact location of the threshold level when event rates are very low. Although there was no statistically significant departure from linearity, our data suggest that there may be a 'basement' level to the graded relation between glycaemia and health risk with potentially no relevant mortality benefit of further lowering glycaemic haemoglobin. Such a notion has been suggested recently for fasting glucose and cardiovascular disease [17]. The basement range found in our study corresponds to the 'normal' range of glycated haemoglobin found in people in the general population who have normal glucose tolerance (mean glycated haemoglobin $5.3 \pm 0.3 \%$ ) [18]. This is compatible with recent suggestions [19] that normal levels of glucose ought not to be associated with increased health risk given the essential requirement for glucose to sustain metabolic activity and life and consequently the tight regulation of blood glucose levels.

The shape of the risk relationship analysis of glycated haemoglobin with cause-specific mortality was similar to that for all-cause mortality, showing that the relationship with all-cause mortality was strongly mediated by cardiovascular mortality. The number of cardiovascular deaths in the lowest category of glycated haemoglobin was low, but previous findings on combined fatal and non-fatal cardio- 
vascular events in the ARIC study and our population, resulting in larger numbers of events, also confirmed an increased risk only for the high range of glycated haemoglobin. Furthermore, we observed a similar shape of association for cancer-related mortality, with an increased risk for increasing glycated haemoglobin levels. Previous studies examining the association of glycated haemoglobin with incident cancer or cancer-related mortality were limited by small numbers of cases, which did not allow reliable risk estimation for the broad range of glycated haemoglobin levels [7, 20-23]. The largest analysis of a case-control set for colorectal cancer within the total EPIC cohort described a significantly increased risk of hyperglycaemia in agreement with our results on mortality from any cancer type [22]. The underlying mechanism remains unclear and exploration is beyond the scope of this study. However, the important finding here is that there was no evidence for an increased risk of low levels of glycated haemoglobin for cancer-related mortality which, next to cardiovascular disease, is the leading cause of mortality in the elderly population.

The mechanistic pathways underlying the relationship between low glycaemia and health risk might differ overall between patients with treated diabetes and nondiabetic individuals. However, recent post hoc analyses of the Action to Control Cardiovascular Risk in Diabetes (ACCORD) trial also suggested that a subgroup of patients with refractory hyperglycaemia accounts for the increased mortality risk observed with the intensive treatment strategy in diabetic patients and not ordinary lower glycaemia [24-26].

The explanations for the different findings of our study compared with the ARIC study and NHANES remain to be determined. In contrast to our homogeneous study population of white Europeans, the design of the ARIC study and NHANES is multi-ethnic, with about $11-22 \%$ blacks included. Blacks have higher levels of glycated haemoglobin than whites, even after correcting for glucose levels [27]. It is unclear whether these racial disparities have relevance for the susceptibility to glycaemia-associated complications. Analyses of NHANES suggested that the increased mortality risk associated with low levels of glycated haemoglobin is partly mediated by liver disease, specifically hepatitis C [3]. We observed much lower levels of markers of liver damage compared with NHANES overall and particularly no increase in these markers in individuals with low glycated haemoglobin. Furthermore, the prevalence of seropositive hepatitis $\mathrm{C}$ assessed in a subset of the UK EPIC cohorts was $0.6 \%$ [28], which is also much lower than in NHANES participants $(2.3 \%)$; this might therefore partly explain the divergent findings for the association between glycated haemoglobin and mortality risk.
The strengths of our study are the long and complete follow-up as well as the large sample size. We had nearly four times more individuals with glycated haemoglobin below 5\% compared with the ARIC study and $10 \%$ more individuals compared with NHANES, which allowed us to estimate the association between glycated haemoglobin and mortality precisely for the range of maximum interest. A limitation of our study is the virtually exclusive inclusion of white Europeans. Thus, our results cannot be generalised to other ethnic groups and our study also cannot contribute to the exploration of ethnic differences in the relation of glycated haemoglobin and health outcome. Furthermore, our analyses are based on a single measurement of glycated haemoglobin at baseline. This could lead to underestimation of the true association due to error resulting from random variability; however, the within-participant variation of glycated haemoglobin in non-diabetic individuals is minimal (coefficient of variation 1.7\%) [29]. Finally, we did not have sufficient events to exclude an increased risk of mortality from specific cancer subtypes in individuals with low glycated haemoglobin. However, it is unlikely that an association with very rare diseases would underlie the findings of the ARIC study and NHANES or be relevant for the clinical use of glycated haemoglobin.

In conclusion, our findings in a large non-diabetic population of white Europeans do not support the concern about increased mortality risk associated with low levels of glycated haemoglobin. Differences in population characteristics including the prevalence of co-morbid conditions might explain the discrepant results across study populations. Further research is needed to explore determinants of low glycated haemoglobin which are concurrently associated with increased mortality.

Acknowledgements We thank all of the participants in this study and the EPIC-Norfolk study staff at the University of Cambridge, Department of Public Health and Primary Care. Supported by grants (to R. Pfister) from the Koeln Fortune Program and the Marga- und Walter-Boll-Stiftung, and by grants (to the EPIC-Norfolk study) from the Medical Research Council UK and Cancer Research UK.

Duality of interest All authors declare that they have no conflicts of interest. The sponsors had no role in the design, data collection, data analysis, data interpretation, or writing or revisions of the report. The corresponding author had full access to all data in the study and had final responsibility to submit for publication.

\section{References}

1. Association AD (2010) Diagnosis and classification of diabetes mellitus. Diabetes Care 33(Suppl 1):S62-S69

2. Selvin E, Steffes MW, Zhu H et al (2010) Glycated hemoglobin, diabetes, and cardiovascular risk in nondiabetic adults. N Engl J Med 362:800-811 
3. Carson AP, Fox CS, McGuire DK et al (2010) Low hemoglobin A1c and risk of all-cause mortality among US adults without diabetes. Circ Cardiovasc Qual Outcomes 3:661-667

4. Currie CJ, Peters JR, Tynan A et al (2010) Survival as a function of $\mathrm{HbA}(1 \mathrm{c})$ in people with type 2 diabetes: a retrospective cohort study. Lancet 375:481-489

5. Gerstein HC, Miller ME, Byington RP et al (2008) Effects of intensive glucose lowering in type 2 diabetes. $\mathrm{N}$ Engl $\mathrm{J}$ Med $358: 2545-2559$

6. Brewer N, Wright CS, Travier N et al (2008) A New Zealand linkage study examining the associations between A1C concentration and mortality. Diabetes Care 31:1144-1149

7. Levitan EB, Liu S, Stampfer MJ et al (2008) HbAlc measured in stored erythrocytes and mortality rate among middle-aged and older women. Diabetologia 51:267-275

8. de Vegt F, Dekker JM, Ruhe HG et al (1999) Hyperglycaemia is associated with all-cause and cardiovascular mortality in the Hoorn population: the Hoorn Study. Diabetologia 42:926-931

9. Barr EL, Boyko EJ, Zimmet PZ, Wolfe R, Tonkin AM, Shaw JE (2009) Continuous relationships between non-diabetic hyperglycaemia and both cardiovascular disease and all-cause mortality: the Australian Diabetes, Obesity, and Lifestyle (AusDiab) Study. Diabetologia 52:415-424

10. Khaw KT, Wareham N, Bingham S, Luben R, Welch A, Day N (2004) Association of hemoglobin A1c with cardiovascular disease and mortality in adults: the European prospective investigation into cancer in Norfolk. Ann Intern Med 141:413420

11. Khaw KT, Wareham N, Luben R et al (2001) Glycated haemoglobin, diabetes, and mortality in men in Norfolk cohort of european prospective investigation of cancer and nutrition (EPIC-Norfolk). BMJ 322:15-18

12. Day N, Oakes S, Luben R et al (1999) EPIC-Norfolk: study design and characteristics of the cohort. European Prospective Investigation of Cancer. Br J Cancer 80(Suppl 1):95-103

13. Khaw KT, Dowsett M, Folkerd E et al (2007) Endogenous testosterone and mortality due to all causes, cardiovascular disease, and cancer in men: European prospective investigation into cancer in Norfolk (EPIC-Norfolk) Prospective Population Study. Circulation 116:2694-2701

14. Firth D (2003) Overcoming the reference category problem in the presentation of statistical models. Sociol Methodol 33:1-18

15. Coutinho M, Gerstein HC, Wang Y, Yusuf S (1999) The relationship between glucose and incident cardiovascular events. A metaregression analysis of published data from 20 studies of 95,783 individuals followed for 12.4 years. Diabetes Care $22: 233-240$
16. Ford ES, Zhao G, Li C (2010) Pre-diabetes and the risk for cardiovascular disease: a systematic review of the evidence. J Am Coll Cardiol 55:1310-1317

17. Sarwar N, Gao P, Seshasai SR et al (2010) Diabetes mellitus, fasting blood glucose concentration, and risk of vascular disease: a collaborative meta-analysis of 102 prospective studies. Lancet 375:2215-2222

18. Van't Riet E, Alssema M, Rijkelijkhuizen JM, Kostense PJ, Nijpels G, Dekker JM (2010) Relationship between A1C and glucose levels in the general Dutch population: the new Hoorn Study. Diabetes Care 33:61-66

19. Gerstein HC (2010) More insights on the dysglycaemiacardiovascular connection. Lancet 375:2195-2196

20. Lin J, Ridker PM, Pradhan A et al (2005) Hemoglobin A1c concentrations and risk of colorectal cancer in women. Cancer Epidemiol Biomark Prev 14:3010-3012

21. Lin J, Ridker PM, Rifai N et al (2006) A prospective study of hemoglobin A1c concentrations and risk of breast cancer in women. Cancer Res 66:2869-2875

22. Rinaldi S, Rohrmann S, Jenab M et al (2008) Glycosylated hemoglobin and risk of colorectal cancer in men and women, the European prospective investigation into cancer and nutrition. Cancer Epidemiol Biomark Prev 17:3108-3115

23. Travier N, Jeffreys M, Brewer N et al (2007) Association between glycosylated hemoglobin and cancer risk: a New Zealand linkage study. Ann Oncol 18:1414-1419

24. Riddle MC, Ambrosius WT, Brillon DJ et al (2010) Epidemiologic relationships between $\mathrm{A} 1 \mathrm{C}$ and all-cause mortality during a median 3.4-year follow-up of glycemic treatment in the ACCORD trial. Diabetes Care 33:983-990

25. Bonds DE, Miller ME, Bergenstal RM et al (2010) The association between symptomatic, severe hypoglycaemia and mortality in type 2 diabetes: retrospective epidemiological analysis of the ACCORD Study. BMJ 340:b4909

26. Calles-Escandon J, Lovato LC, Simons-Morton DG et al (2010) Effect of intensive compared with standard glycemia treatment strategies on mortality by baseline subgroup characteristics: the Action to Control Cardiovascular Risk in Diabetes (ACCORD) trial. Diabetes Care 33:721-727

27. Ziemer DC, Kolm P, Weintraub WS et al (2010) Glucoseindependent, black-white differences in hemoglobin A1c levels: a cross-sectional analysis of 2 studies. Ann Intern Med 152:770-777

28. Franceschi S, Lise M, Trepo $C$ et al (2011) Infection with hepatitis $\mathrm{B}$ and $\mathrm{C}$ viruses and risk of lymphoid malignancies in the European Prospective Investigation into Cancer and Nutrition (EPIC). Cancer Epidemiol Biomark Prev 20:208-214

29. Rohlfing C, Wiedmeyer HM, Little R et al (2002) Biological variation of glycohemoglobin. Clin Chem 48:1116-1118 